\title{
FISSION SEQUENCE AND EQUILIBRIUM MODELS OF RIGIDITY ROTATING POLYTROPES*
}

\author{
IZUMI HACHISU and YOSHIHARU ERIGUCHI** \\ Department of Aeronautical Engineering, Kyoto University, Kyoto, Japan
}

(Received 4 June, 1983)

\begin{abstract}
Equilibrium sequences of self-gravitating and polytropic stars including binary stars are computed for various polytropic indexes. We find a fission sequence from an ellipsoidal configuration to a binary by way of dumb-bell equilibrium.
\end{abstract}

\section{3D Hydrostatic Equilibriums}

It is required to obtain accurate equilibrium models of rotating stars including binary stars from the following points of view: (1) reexamination of the fission theory; (2) checking the results of three-dimensional (3D) hydrodynamical calculation; (3) proposing proper model of mass-exchange or mass-losing close binary systems.

Recently, we have developed methods for obtaining 3D hydrostatic equilibrium of rotating polytropes, and succeeded in calculating various sequences. In this paper we will summarize our results and discuss the physical nature of the sequences.

\section{Equilibrium Sequences}

\subsection{INCOMPRESSIBLE CASE (POLYTROPIC INDEX $n=0$ )}

As many bifurcation points have been found on the Maclaurin and the Jacobi sequences, the calculations are started from these points and the equilibriums are obtained step by step. These sequences are shown in Figure 1.

(A) Pear-Shaped: this bifurcates from the Jacobi sequence but it terminates shortly by mass-shedding (this denotes that the gravity balances with or is less than the centrifugal force at the surface). The shapes just before mass-shedding are shown in Figure 2.

(B) Dumb-Bell: this also bifurcates from the Jacobi sequence and continues to binary sequence with mass ratio $q=1$. This is a fission sequence.

(C) Binary sequences: the less massive component necessarily fills up its Roche lobe first and the sequences terminate.

\footnotetext{
* Paper presented at the Lembang-Bamberg IAU Colloquium No. 80 on 'Double Stars: Physical Properties and Generic Relations', held at Bandung, Indonesia, 3-7 June, 1983.

** Department of Earth Science and Astronomy, College of Arts and Sciences, University of Tokyo, Japan.
} 


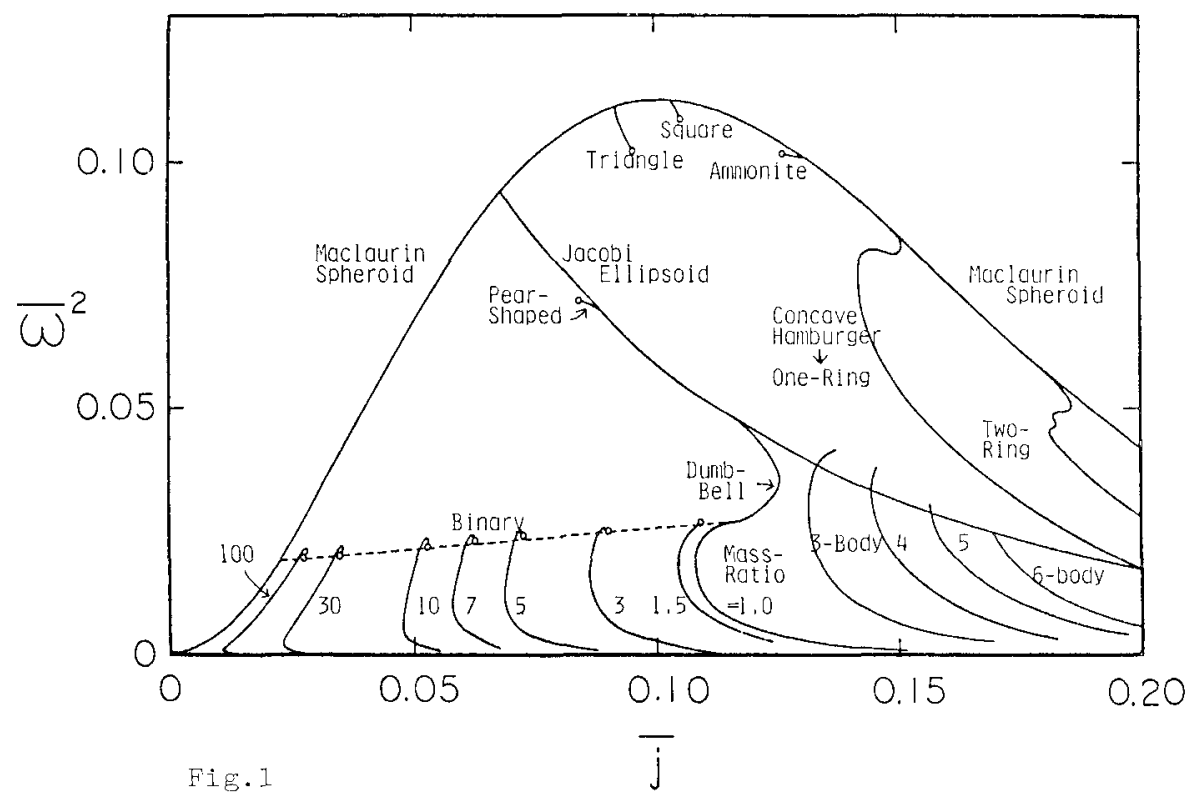

Fig. 1. Angular velocity $\bar{\omega}^{2}$-angular momentum $\bar{j}$ diagram.
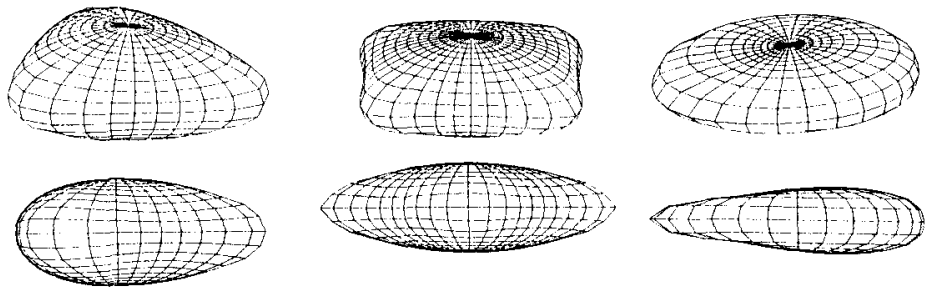

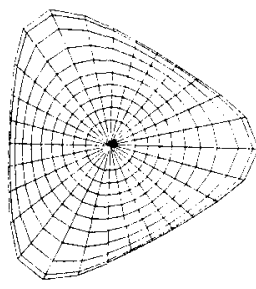

Triangle

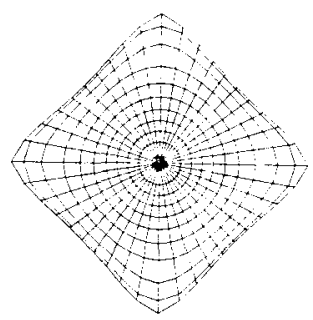

Square

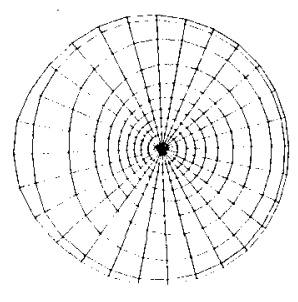

Ammonite

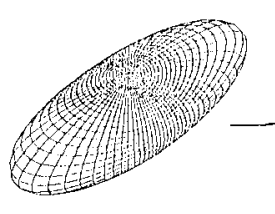

Jacobi

Ellipsoid

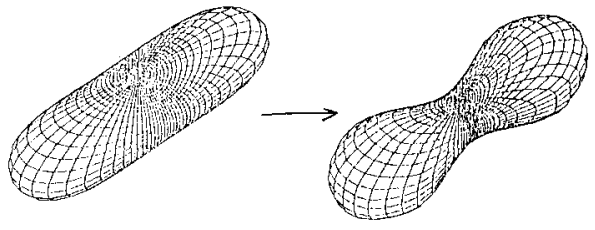

Dumb-Bell

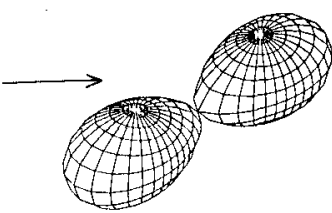

Binary

Fig. 2. 

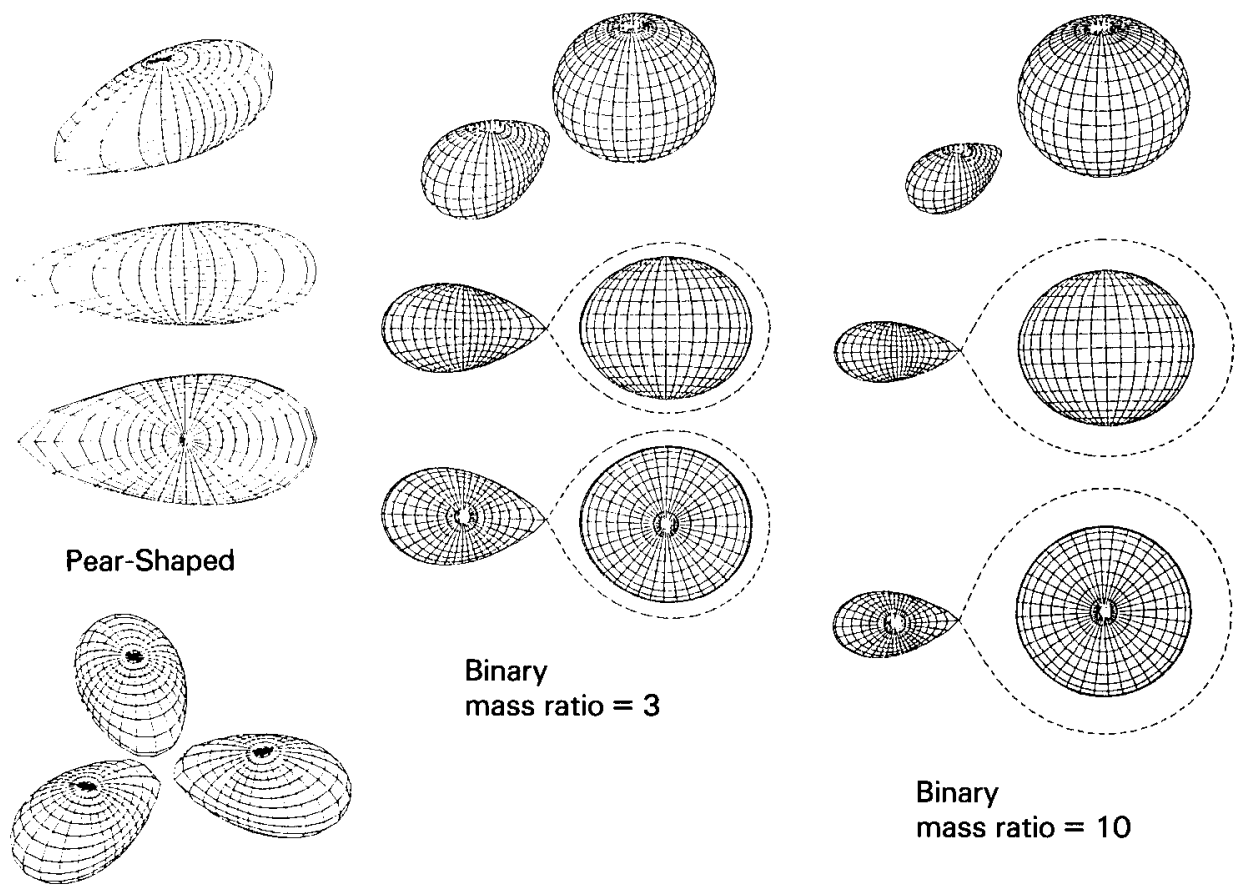

$$
\begin{aligned}
& \text { Binary } \\
& \text { mass ratio }=3
\end{aligned}
$$

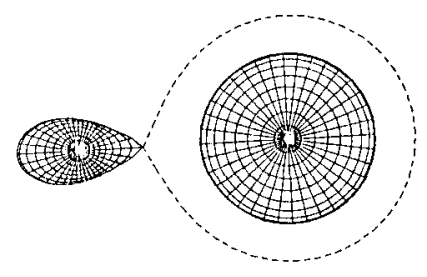

$$
\begin{aligned}
& \text { Binary } \\
& \text { mass ratio }=10
\end{aligned}
$$

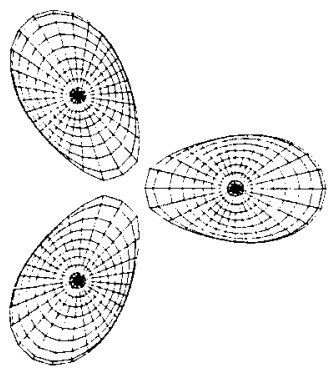

3-Body Equilibrium
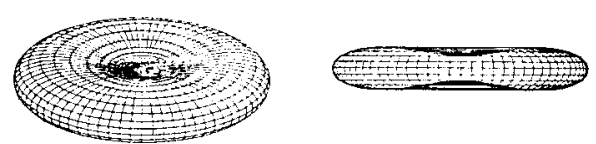

\section{Concave Hamburger}

Fig. 2. (continued)

(D) Triangle: this bifurcates from the Maclaurin sequence. This sequence also shortly terminates because of mass-shedding.

(E) Square.

(F) Ammonite.

(G) Concave Hamburger: this also bifurcates from the Maclaurin sequence and continues to the Dyson-Wong toroid. We call this sequence One-Ring sequence.

(H) Two-Ring: this bifurcates from the Maclaurin sequence.

(J) Multi-Body: we can calculate multi-body sequences.

\subsection{Compressible CASE (POLYTROPIC INDEX $n \neq 0$ )}

Various sequences with various polytropic indexes have been computed. However, there are no thorough sequences thoroughly from spheroid-like configuration to binary 


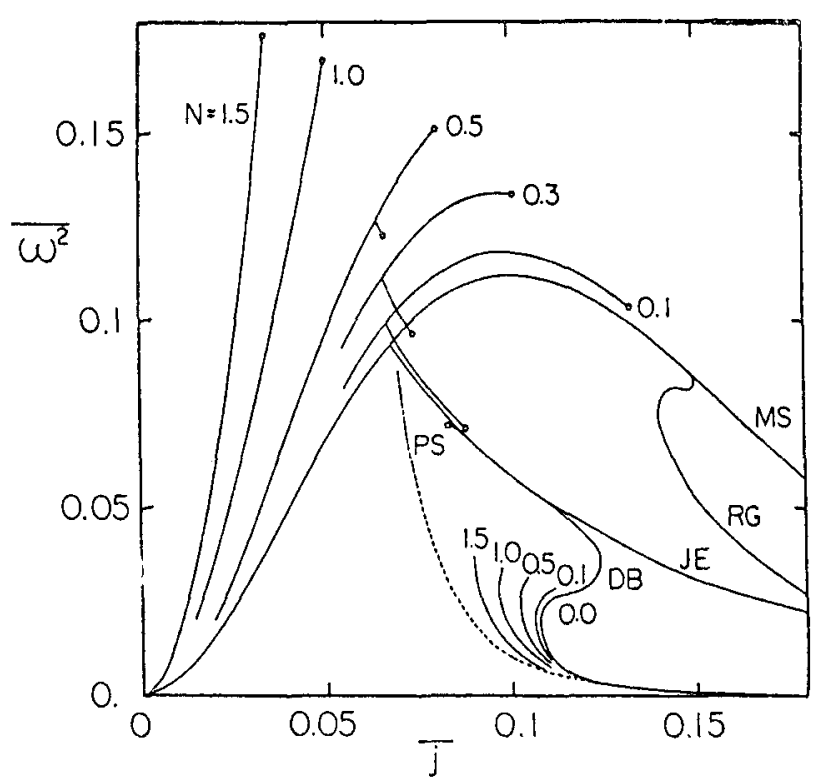

Fig. 3. $\bar{\omega}^{2}-\bar{j}$ diagram. MS: Maclaurin sequence. JE: Jacobi sequence. DB: Dumb-Bell sequence. PS: Pear-Shaped sequence. RG: One-Ring sequence. And two-point mass sequence (dotted) is added. Open circle denotes the point where mass-shedding occurs.

configuration because of mass-shedding as seen in Figure 3. Five binary sequences with polytropic indexes $N=0,0.1,0.5,1.0$, and 1.5 have been computed to the contact stage.

\section{References}

(A) Dumb-Bell: Pear-Shaped;

Eriguchi, Y., Hachisu, I., and Sugimoto, D.: 1982, Prog. Theor. Phys. 67, 1068.

Hachisu, I. and Eriguchi, Y.: 1982, Prog. Theor. Phys. 68, 206.

(B) Binary;

Hachisu, I. and Eriguchi, Y.: 1983a, submitted to Publ. Astron. Soc. Japan (Compressible case).

Hachisu, I. and Eriguchi, Y.: 1983b, submitted to Publ. Astron. Soc. Japan (Incompressible case).

Hachisu, I. and Eriguchi, Y.: 1983c, submitted to Publ. Astron. Soc. Japan (Incompressible case, planet and satellite system).

(C) Triangle: Square: Ammonite: Two-Ring;

Eriguchi, Y. and Hachisu, I.: 1982, Prog. Theor. Phys. 67, 844.

Eriguchi, Y. and Hachisu, I.: 1983, Prog. Theor. Phys. 69, 1131.

(D) Concave Hamburger: One-Ring;

Eriguchi, Y. and Sugimoto, D.: 1981, Prog. Theor. Phys. 65, 1870 (Incompressible case).

Hachisu, I. and Eriguchi, Y.: 1982, Prog. Theor. Phys. 68, 191 (Compressible case).

(E) Multi-Body;

Eriguchi, Y. and Hachisu, I.: 1983, submitted to Prog. Theor. Phys. 